\title{
ASC data processing center for RadioAstron project
}

\author{
Andrey Andrianov ${ }^{1}$ \\ Astro Space Center of Lebedev Physical Institute \\ 84/32 Profsoyuznaya Street, Moscow, Russian Federation \\ E-mail: andrianeasc.rssi.ru
}

\section{Marina Shatskaya, Nikolai Fedorov, Igor Guirin, Vladimir Kostenko, Sergey Likhachev, Sergey Seliverstov \\ Astro Space Center of Lebedev Physical Institute \\ 84/32 Profsoyuznaya Street, Moscow, Russian Federation \\ E-mail: mshatskeasc.rssi.ru, Nikolai_Fedoroveasc.rssi.ru, \\ igirineasc.rssi.ru, vkostenkodasc.rssi.ru, slikhacheasc.rssi.ru, \\ selivercasc.rssi.ru}

A scientific data processing center was organized specifically for the RadioAstron project. The main goals of the center are acquisition and storage of different types of information along with data processing. This paper reviews the capabilities and performances of the processing center.

11th European VLBI Network Symposium \& Users Meeting

October 9-12, 2012

Bordeaux, France

${ }^{1}$ Speaker 


\section{Introduction}

RadioAstron $^{2}$ is an international space VLBI mission led by the Astro Space Center of the Lebedev Physical Institute, located in Moscow (Russian Federation). The main payload of the mission is a $10 \mathrm{~m}$ space radio telescope designed by Lavochkin Association of Roscosmos Russia State Agency. The project goal is to create, together with a network of ground based radio telescopes, a huge ground to space interferometer with baselines up to $350000 \mathrm{~km}$, to obtain images, positions and movements of various objects in the Universe with extremely high angular resolution (up to a few $10^{-6} \mathrm{arcsec}$ ). After its launch on 18 July 2012 (Fig. 1), the RadioAstron mission started such investigations, observing a broad range of radio frequencies.

In order to provide the necessary support to the RadioAstron project, a Scientific Data Processing Center (SDPC) was specifically organized. SDPC is a fail-safe complex with a centralized system of interconnected software and hardware components and organizational procedures designed for reliable data storage and processing, along with delivery of services and applications. This system also possesses a high degree of virtualization of its resources. The main tasks performed by SDPC are:

- data acquisition and storage in a specialized data repository;

- delivery of services to users;

- data processing on a high-performance computer complex.

\section{Computer Complex}

The block diagram of the computer complex designed at the Astro Space Center for the RadioAstron project is shown in Fig. 2. The complex includes the following components:

- control unit;

- computer cluster;

- data repository with a capacity of $200 \mathrm{~TB}$;

- backup system on magnetic tapes (32 TB);

- 24 TB redundant storage system at the Pushchino Radio Astronomy Observatory;

- archive of data on magnetic tapes and HDD (400 TB);

- web and ftp servers;

- networks for managing and transmitting data.

The cluster (main component of the complex) is a group of computers connected with highspeed communication channels. It is an integrated hardware resource including one control and five computing servers assembled in a common rack. The servers are connected to each other by two networks, one of which (with a speed of $10 \mathrm{~Gb} / \mathrm{s}$ ) is used for transferring MPI traffic, while the other (Gigabite Ethernet) is used to manage the storage system. The latter is also used to access remote desktop servers in the cluster through the control unit.

The data storage system consists of a $200 \mathrm{~TB}$ repository, a redundant system of $24 \mathrm{~TB}$ capacity, backup systems (tape library) with 32 TB capacity, and an archive of 400 TB capacity. Fail-safety of the storage system is achieved by using two redundant power supplies with hot-

\footnotetext{
${ }^{2}$ See http://www.asc.rssi.ru/index.html.
} 
swap ability and three cooling fans, also with hot-swap ability. Storage reliability is achieved by using RAID6. Two disks are allocated to check sums; double sums are calculated using different algorithms. In this scheme, disk arrays are used as single logical disks. The disk arrays have additional capacity permitting restoration of data in case of sudden failures. The tape library "Tandberg Storage Library T40" permits prompt file backup. Its purpose is the longterm storage of the raw data and of rarely requested data after processing. In the case of failure of any component of the computer complex or its disconnection, a 24 TB data storage system installed in Pushchino can take over in order to prevent data loss.

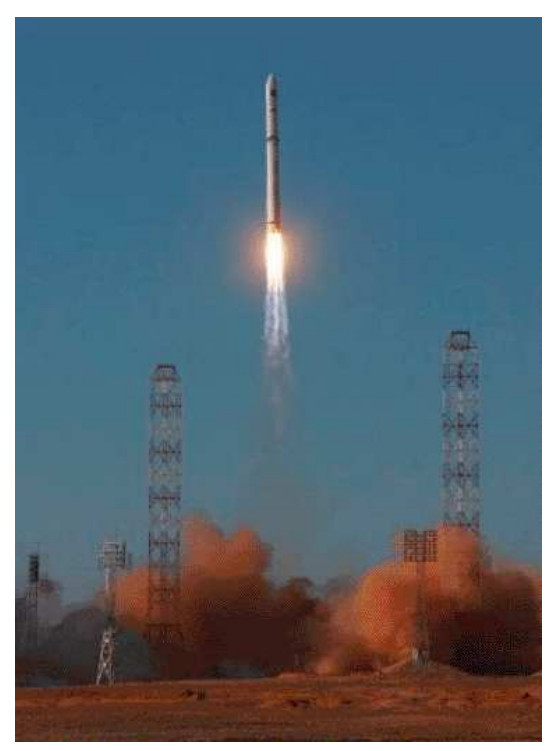

Figure 1. Launch of the RadioAstron mission on 18 July 2012.

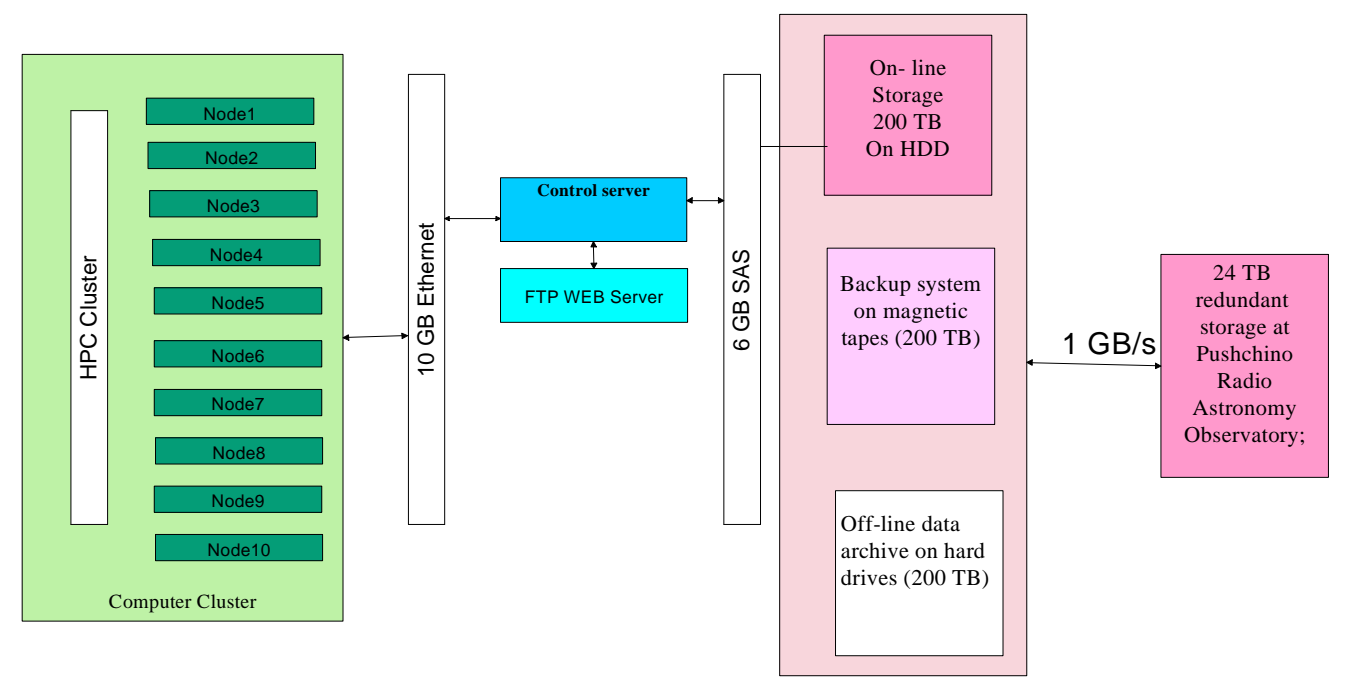

Figure 2. Block diagram of the computer cluster. 


\section{Network infrastructure of the processing center and communication channels}

One of the aims of the processing center is to organize the flow of information and its exchange. SDPC receives information from different places such as the tracking station, the mission control center, ground-based telescopes, the ballistic center, and tracking headquarters (Fig. 3).

Transmission of large amounts of data over long distances and delivery of information in on-line mode requires high-speed communication channels. To this end, appropriate equipment based on optical links have been tested and installed. Such high-speed links have been organized to connect SDPC in Moscow, the tracking station in Pushchino and tracking headquarters in Lavochkin Association. The tracking station and SDPC are connected through Gigabit Ethernet for transmission of scientific data from the space telescope. At present, SDPC and tracking headquarters are connected through a 2 Mbit/s link.

The primary transmitted information is scientific data. Data from ground-based radio telescopes (see participating telescopes in Fig. 4) is transmitted over internet using proper links and agreed protocols. For higher efficiency, we first increased our connection speed to $70 \mathrm{Mbit} / \mathrm{s}$ but this was not enough and we had to increase it further to $100 \mathrm{Mbit} / \mathrm{s}$, keeping an opportunity for expansion. At present, this transmission speed remains the limiting factor in our system. It depends on the state of internet and of links to specific telescopes. For places where there are no fast communication channels, we obtain information from hard drives. The different methods used for delivery of information are summarized in Fig.5.

Another way for rapidly exchanging information is through a ftp-server by which we exchange data between the center of experiment planning, the tracking station, the ballistic center, and tracking headquarters. For this purpose, we created a specific structure of directories and files. Access is restricted to only authorized users in accordance with their rights. Our ftpserver contains information about the predicted and reconstructed orbit, the latest version of the plan of experiment, telemetry, software and other information.

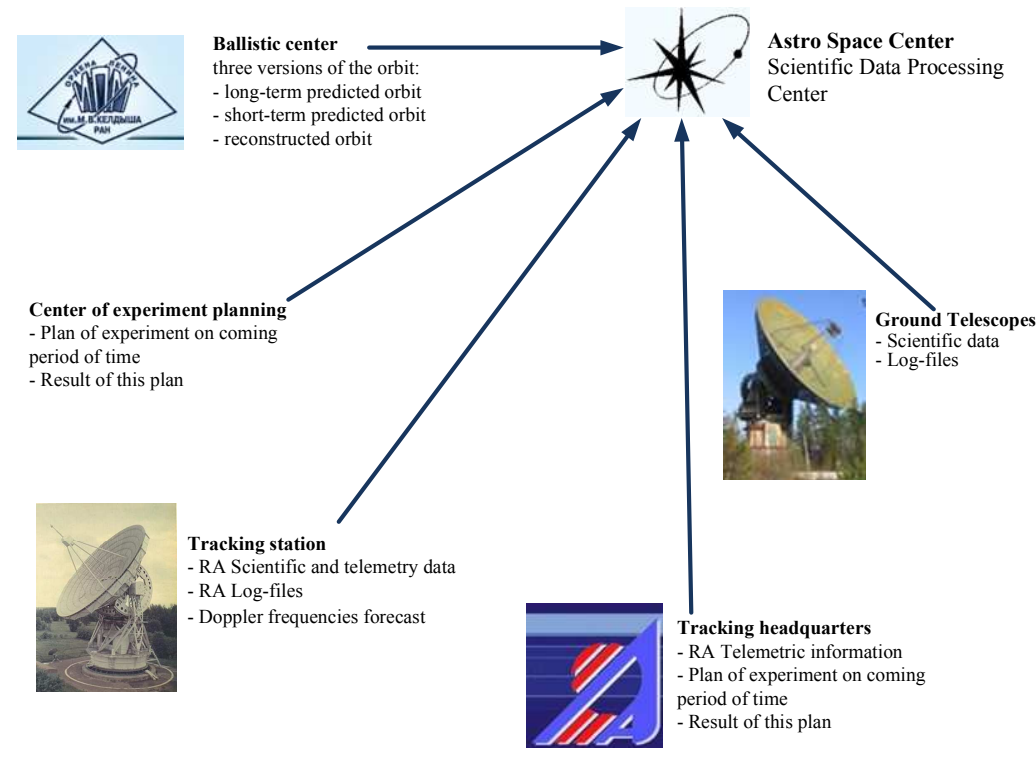

Figure 3. Flow of information between the various components of the mission. 


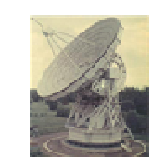

Pushing

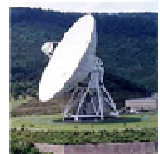

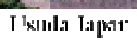

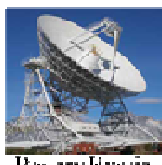

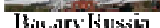
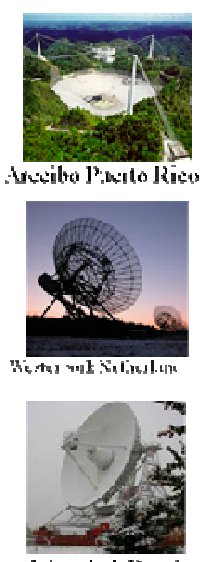

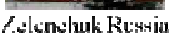

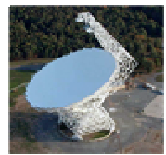

tirtTS
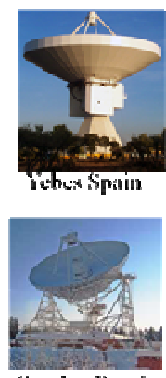

Shilline Ruscia

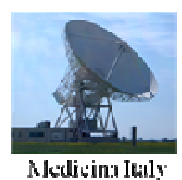

Meditina Italy

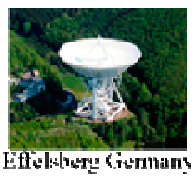

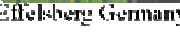

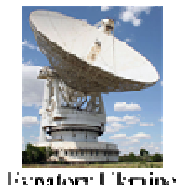

Inpahror I'knim

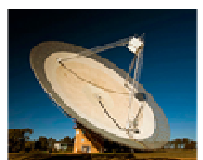

[3ates Lustralat
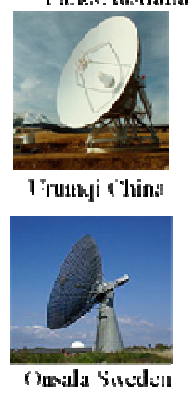
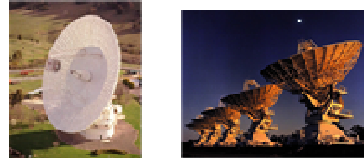

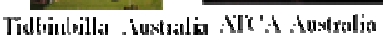

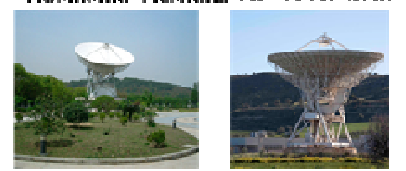

Shanglui i.'lün

Sit: Italy
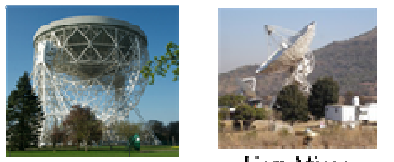

I"ar: Utiltoul

Figure 4. Ground-based radio telescopes observing jointly with RadioAstron.

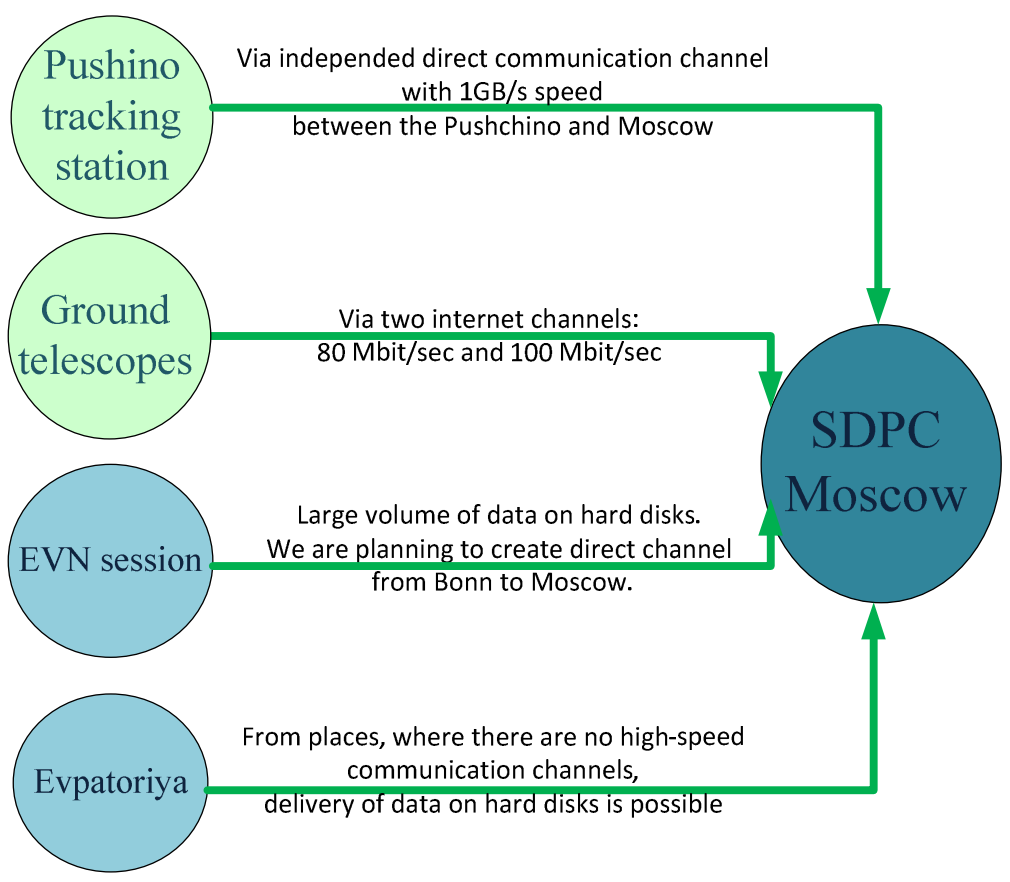

Figure 5. Delivery of information to SDPC.

\section{Conditioning and reliability}

Modern high-tech computing and telecommunication equipment is sensitive to very small changes in the environment. For ensuring normal operation, maintaining strictly determined temperature and humidity level is mandatory. The computer room was thus equipped with conditioning and ventilating systems that are connected according to the scheme $\mathrm{N}+1$. In case of failure of one conditioner, another one is automatically turned on. Additionally, a no-break power supply system is organized for servers on the basis of APC, which excludes data loss in case of power failure or jumps, and disconnection of one or more power cables. 


\section{Conclusion}

The infrastructure of the ASC Data Processing Center has been designed to be fully adequate to the data processing requirements of the RadioAstron mission. Its performances have been confirmed during Fringe Search and Early Science Program in flight operations. 